\title{
Carl Menger, fondateur de l'« école autrichienne », en son temps et dans sa ville
}

Carl Menger, Gründer der österreichischen Schule, zu seiner Zeit und in seiner Stadt

Carl Menger, founder of the Austrian school, in the context of his times in his city

\section{Herta Mayerhofer}

\section{(Q) OpenEdition}

\section{Journals}

Édition électronique

URL : https://journals.openedition.org/austriaca/1015

DOI : 10.4000/austriaca.1015

ISSN : 2729-0603

\section{Éditeur}

Presses universitaires de Rouen et du Havre

\section{Édition imprimée}

Date de publication : 1 juin 2020

ISBN : 979-10-240-1492-0

ISSN : 0396-4590

\section{Référence électronique}

Herta Mayerhofer, "Carl Menger, fondateur de l'« école autrichienne », en son temps et dans sa ville », Austriaca [En ligne], 90 | 2020, mis en ligne le 01 juin 2021, consulté le 03 octobre 2022. URL : http:// journals.openedition.org/austriaca/1015; DOI : https://doi.org/10.4000/austriaca.1015

Ce document a été généré automatiquement le 3 octobre 2022.

Tous droits réservés 


\title{
Carl Menger, fondateur de l'« école autrichienne », en son temps et dans sa ville
}

\author{
Carl Menger, Gründer der österreichischen Schule, zu seiner Zeit und in seiner \\ Stadt \\ Carl Menger, founder of the Austrian school, in the context of his times in his \\ city
}

Herta Mayerhofer

\section{Introduction}

1 À l'époque de Carl Menger (1840-1921), Vienne déployait toute sa splendeur dans les domaines de l'architecture, de la peinture, de la littérature et de la musique. Pour préparer l'agrandissement et la modernisation de la capitale, l'empereur FrançoisJoseph fit abattre la vieille enceinte afin de percer un large boulevard circulaire, la Ringstrasse, qui devait bientôt être bordée de grands palais publics et privés. Artistes, scientifiques et chercheurs de renom, parmi lesquels quelques représentants de la minorité juive comme Arthur Schnitzler, Gustav Mahler et Sigmund Freud, contribuèrent à rendre possible (malgré l'antisémitisme latent) le bouillonnement artistique et intellectuel qui faisait de Vienne la capitale culturelle européenne de la fin du XIX siècle.

2 À la même époque, les ouvriers travaillant dans l'industrie et les paysans vivant dans les régions périphériques de l'Empire souffraient énormément ou s'apprêtaient à se révolter. Malgré des efforts de réforme, souvent entrepris sans conviction ni esprit de décision, le mythe habsbourgeois de la coexistence harmonieuse entre les peuples de langues et nations différentes se brisa en 1914 à l'occasion des atrocités de la première guerre mondiale. 
3 Durant le dernier tiers du $\mathrm{XIX}^{\mathrm{e}}$ siècle, les idées économiques à la base des tentatives de réforme avaient éclos. Celles de Menger concernant la promotion d'une théorie économique pure demeuraient vivaces. Comment sont-elles nées ? D’où proviennentelles?

4 Menger est né en 1840 au sein d'une famille aisée de Galicie. Il accomplit ses études supérieures à Vienne, à Prague et à Cracovie ${ }^{1}$. Une fois en possession de ses diplômes, Menger écrit des articles économiques et politiques pour la gazette de Lemberg (Lvov), puis il se rend à Vienne. Là, il cofonde le quotidien Wiener Tagblatt, qui doit devenir le Neues Wiener Tagblatt. La qualité de ses articles et ses connaissances économiques, obtenues notamment par son travail d'analyste des cours de la Bourse, ne restent pas inaperçues des spécialistes, et Menger devient, à partir de 1869, membre du « bureau de presse auprès de la présidence du Conseil des ministres autrichien ", dont l'influence sur l'administration était alors de fait considérable.

Après avoir obtenu la qualification nécessaire, Menger se tourne vers la carrière académique : il est nommé Extraordinarius (professeur assistant) en 1873, puis, en 1879, Ordinarius (professeur titulaire) occupant la chaire de théorie économique à l'université de Vienne. Menger sépare strictement ses activités à l'université : d'une part, il enseigne sa nouvelle théorie de la valeur et sa fonction à la Cour ; d'autre part, il est le précepteur du jeune prince héritier Rudolf de 1876 à 1878. Il conserve par la suite une relation amicale avec l'héritier présomptif de la couronne impériale, qu'un destin tragique attend. Parallèlement à son activité de professeur, Menger continue son travail pour les journaux et il collabore avec les cabinets ministériels. Il est conseiller principal de la réforme monétaire (Valutareform) de 1892.

6 Les expériences personnelles de Menger durant son enfance en Galicie, ses études universitaires à Prague, à Vienne et à Cracovie, ses activités dans le domaine de la science, dans l'appareil de la bureaucratie impériale et dans les milieux des affaires et de la finance comme dans ceux du journalisme donnent à Menger une position exceptionnelle d'observateur des réalités de l'empire finissant. Il peut suivre au plus près les événements de son époque et il se trouve ainsi participer du mouvement large de modernisation dans les arts, les sciences, l'université même, dans cette ville de Vienne qui était devenue la sienne. Nous tenterons ici de présenter tout cela : l'homme dans sa ville et dans son époque. Menger élabore ainsi sa propre théorie innovante de la valeur sur fond d'antihistoricisme et de subjectivisme (section 2). Il prend position à l'égard des thèses de la social-démocratie (section 3 ) ; il comprend les problèmes du nationalisme (section 4); il tente enfin de remédier aux déséquilibres entre les régions (section 5).

\section{Vienne, ville florissante, au centre de la vie de Menger}

7 Tel un long chant d'adieu, Vienne lança, à la veille de disparaître comme capitale impériale à l'issue de la première guerre mondiale, une extraordinaire clameur. L'épanouissement culturel et artistique de la période dite «Vienne 1900 » ou «Vienne fin de siècle » reste d'un éclat incomparable ${ }^{2}$. Ce rayonnement concerna l'architecture, les arts plastiques, la musique, la littérature, ou encore, dans le champ du savoir, la naissance de la psychanalyse et, parmi les sciences sociales, l'essor d'une nouvelle théorie de l'économie théorique pure qui relève d'une nouvelle approche de la connaissance et dont fait éminemment partie la découverte du « marginalisme » par 
Menger. Il faut replacer la théorie de Menger au sein de cette efflorescence de questions posées à des doctrines qui provenaient d'autres domaines et qu'il devait rencontrer dans le sien sous la forme de l'historicisme qu'il combattit en économie, et qui était aussi au cœur des débats artistiques.

\section{Historicisme et subjectivité : pour ou contre ? Le débat venu de l'architecture et des arts}

8 Le 20 décembre 1857, l'empereur François-Joseph décrète l'instauration d'une commission chargée d'organiser l'expansion urbaine de Vienne. Les fortifications militaires et le glacis qui avaient autrefois protégé la ville des incursions turques sont démolis pour construire la Ringstrasse, une avenue décorée de bâtiments d'un luxe extraordinaire. Sans développer de style architectural spécifique, chaque bâtiment emprunte celui qui est historiquement le plus à même de traduire la fonction pour laquelle il est prévu.

9 L'architecte Heinrich Ferstel construit d'abord la Votivkirche (l'église votive) en style néo-gothique : un monument au patriotisme et à la dévotion du peuple autrichien pour la dynastie impériale, elle commémore l'attentat manqué contre François-Joseph. L'opéra d'État de Vienne (Wiener Staatsoper), premier des grands projets du Ring, fut construit de 1861 à 1869 par les architectes August von Siccardsburg et Eduard van der Nüll dans un style néo-renaissance, aux contours flous. Quatre édifices publics représentent des valeurs dites « libérales » : le parlementarisme (avec le Parlement, ou Reichsrat), l'autonomie municipale (avec l'hôtel de ville ou Rathaus), le théâtre comme lieu de rencontre réunissant les aristocrates, le clergé et les gens du peuple (avec le théâtre municipal, ou Burgtheater) et la libre indépendance de la pensée dans les sciences (avec l'université). Totalement indépendante des bâtiments voisins - le Parlement et le Rathaus -, et séparée d'eux par un parc, l'université est due à l'architecte Ferstel qui s'est rendu en Italie, berceau de l'humanisme moderne, et a étudié les styles des universités très anciennes de Bologne, de Padoue et de Rome. Pour souligner un lien de nature historique entre elles et le rationalisme moderne, Ferstel choisit le style néo-Renaissance pour édifier cette «citadelle du rationalisme laïc ». C'est dans cette université que le portrait de Menger figure encore aujourd'hui sur un bas-relief en mémoire du détenteur de la chaire de sciences économiques, alors encore pour un temps appelée du vieux nom de Kameralwissenschaften.

10 Le Ring compte par ailleurs, tout autour de la Hofburg impériale, des édifices en divers styles. Les aristocrates, les banquiers et les industriels y construisent leurs propres palais (les palais Ephrussi, Epstein, Lieben-Auspitz, Wertheim, Todesco). D'autres architectes orientent cependant leurs efforts à l'encontre de cet historicisme qui prévaut dans les bâtiments officiels. Ils veulent ainsi répondre aux besoins de leur temps. Adolf Loos dénonce un mensonge culturel dans l'historicisme et les fantaisies archaïsantes de la Ringstrasse. Il s'oppose farouchement à une ornementation devenue selon lui inutile à l'époque contemporaine ${ }^{3}$. Divers groupes, qui se désignent sous le nom Die Jungen (les Jeunes), s'engagent dans un processus de transformation subversive des traditions. Ces mouvements nouveaux s'imposent dans toutes les sphères culturelles: la littérature, l'architecture et la peinture : c'est le Jung Wien (Jeune Vienne) dans le monde littéraire, et le Jugendstil comme mouvement moderniste dans les beaux-arts, embrassant toutes les disciplines en cette fin de XIX ${ }^{e}$ siècle. 
11 Le signe qui ne trompe pas dans ce vaste courant d'innovation consiste dans le rejet d'un historicisme vécu comme inauthentique. Nous en viendrons plus loin aux sciences, mais ce trait était prégnant dans l'esprit des Viennois du temps, qu'ils fussent enthousiasmés ou (plus souvent) choqués par les propositions modernistes. L'architecte Otto Wagner voulait abandonner les valeurs du passé au profit d'une civilisation urbaine organisée en fonction des impératifs de la raison, et il esquissa un plan de reconstruction de Vienne à neuf, sur un schéma fonctionnaliste moderniste. Les bâtiments qu'il prévoyait étaient construits non à des fins de représentation (comme ceux de l'historicisme), mais pour le bien-être de leurs occupants ou de leurs visiteurs : ainsi, la Postsparkasse (Caisse d'épargne de la poste austro-hongroise), l'Église de l'hôpital Steinhof et la Länderbank (banque commerciale indépendante de Vienne). Si les façades de la Postsparkasse sont habillées de plaques servant de décor, les murs intérieurs en plaques de verre visent à rendre l'atmosphère agréable et l'église de l'hôpital Steinhof laisse la lumière entrer à flots. Aujourd'hui l'œuvre d'Otto Wagner fait partie des grandes œuvres classiques du Jugendstil viennois.

Dans les arts plastiques, la lutte contre l'historicisme un temps triomphant s'illustre dans l'évolution de l'activité artistique de Gustav Klimt, dont la vie se divise en plusieurs périodes: d'abord au service de la culture bourgeoise de la Ringstrasse, le jeune Klimt connaît l'admiration du public avec ses peintures historiques pour le Burgtheater dont il décore le plafond du grand escalier intérieur. Au Musée de l'art, il peint le hall d'accueil principal et, pour l'université, trois plafonds du hall d'honneur. Mais quand, dans les années 1870, la contestation "libérale " s'infiltre au même moment dans les arts et dans plusieurs sciences, Klimt prend la tête de la révolte dans les arts plastiques en suscitant le Jugendstil anti-éclectique et anti-historisant.

Sur d'autres principes, et autour de Klimt, des architectes (Josef Maria Olbrich, Josef Hoffmann et Kolo Moser) ainsi que des artistes forment le noyau d'une avant-garde dite « sécessionniste ». Contre la politique d'exposition de l'association officielle des artistes (Künstlergenossenschaft), ces dissidents revendiquent le droit de se livrer à une création " proprement artistique » et, afin de disposer d'un lieu d'exposition pour leurs œuvres, Olbrich dresse les plans de la "maison de la Sécession », sur la forme d'un temple de l'art nouveau. Ses murs nus et blancs, son escalier somptueux donnent un air de dignité païenne à la revendication affichée à son entrée comme devise : « À chaque époque son art, à l'art sa liberté ».

14 Une autre tendance imprègne l'Association des artistes plasticiens d'Autriche (née en mai 1897), alors qu'elle emménage dans sa «nouvelle maison ». C'est, outre la révolte contre le passé (illustrée par l'affiche de Klimt ayant pour thème le combat de Thésée contre le Minotaure pour la libération d'Athènes), la revendication d'un style de plus en plus empreint d'introversion et de subjectivité (c'est dans ce cadre qu'il faut saisir les tableaux de la période dite "dorée ", comme le très fameux Portrait d'Adele Bloch-Bauer, dit «Le Baiser ») : le sujet l'emporte désormais sur l'histoire, à tous égards - or cette leçon devait valoir plus largement.

15 Toujours dans les arts, on peut retrouver les effets de ce bouleversement subjectiviste dans les musiques d'Anton Bruckner, de Gustav Mahler, d'Arnold Schönberg ou d'Alban Berg. Ils composent une musique différente qui cependant, trop savante peut-être, devait ennuyer les couches aisées qui préféraient le style de la «musique légère » de la famille Strauss. Tandis que Mahler, admirateur inconditionnel de Richard Wagner, s'attelle à réaliser l'idéal wagnérien de l'œuvre d'art totale (Gesamtkunstwerk) en 
réformant l'opéra, ses élèves Anton von Webern et Berg fondent «la seconde école de Vienne » (die Zweite Wiener Schule) avec Schönberg qui est quant à lui à l'origine d'une rupture historique avec la musique dodécaphonique. Pendant ce temps, l'opérette viennoise donne la vision opposée d'un monde enchanté, plongé dans son confort. Dans Die Fledermaus, Johann Strauss fils fait chanter le vers devenu célèbre :

Glücklich ist, wer vergisst, was nicht mehr zu ändern ist.

Comme la critique et le public viennois continuent de se montrer hostiles à l'innovation apportée en musique, celle-ci rencontre une influence durable aux États-Unis, où Schönberg émigre en 1933. Les troubles historiques vident la ville de nombre de ses inventeurs, artistes et innovateurs en tous genres: ceux-ci doivent fuir une Vienne insouciante de l'abîme dans lequel elle se précipite et incapable de saisir l'innovation que ses propres écoles de pensée engendrent. Cela vaut également ailleurs que dans les arts: c'est en parallèle l'histoire de certaines sciences sociales, avant tout la psychanalyse et l'école autrichienne d'économie.

\section{Nouvelles théories de la connaissance : de la littérature à la psychanalyse, de la politique aux sciences sociales et économiques}

17 Peut-être plus que n'importe quelle autre ville à n'importe quelle autre époque, la "Vienne fin de siècle » mêla la joie de vivre et l'amertume, l'ouverture au monde et l'intolérance, l'érotisme et la morbidité. Ce mélange détonant contribue sans doute à la séduction qu'elle produit encore et toujours : les contraires s'y trouvaient étroitement liés.

18 Jung Wien, le mouvement littéraire qui, autour de 1890, combattait le moralisme de la littérature $\mathrm{du} \mathrm{XIX}^{\mathrm{e}}$ siècle réclamait plus de vérité sociologique et psychologique, en particulier dans le domaine sexuel. Or les familles des écrivains de Jung Wien faisaient partie des couches les plus conservatrices de la «bonne société » viennoise : déçues de l'agitation causée par les crises à répétition (notamment celle née de la grave crise économique de 1873), ainsi que par les conflits sempiternels sur les questions nationales et sociales, ces couches de la population souhaitaient pour leurs fils un avenir indépendant de la politique. Les pères visaient moins à préparer leurs fils à une activité professionnelle qu'à développer en eux le plaisir esthétique et l'acquisition passive d'un goût certain pour les richesses culturelles qui les entouraient. Les auteurs Hugo von Hofmannsthal, Arthur Schnitzler, Leopold Andrian-Werburg et Richard BeerHofmann peignaient cela. Dans La Mort du Titien, Hofmannsthal montre les protagonistes glissant d'une atmosphère stylée et opulente vers le sentiment de l'agonie et de la mort, comme l'on passe de la réalité au rêve.

19 C'est en particulier dans l'œuvre romanesque et théâtrale de Schnitzler que les états d'âme de cette Vienne fin de siècle se trouvent disséqués. Cette approche rencontre un savoir tout à fait neuf. En effet, l'irruption de l'inconscient en littérature et dans les travaux de Sigmund Freud, qui a été l'élève du neuropsychiatre Charcot, à l'hôpital Sainte-Anne de Paris, ouvre une nouvelle ère dans la conception des maladies de l'esprit. Si L'interprétation des rêves (Die Traumdeutung) de Sigmund Freud définit le rêve comme l'accomplissement d'un désir refoulé, la théorie dépasse de loin l'étiologie sexuelle des névroses. Elle ouvre un chapitre dans la théorie de la connaissance, comme dans la discussion de la notion même de ce qu'est une science. Bien plus tard, un autre enfant de Vienne, Karl Popper, n'hésite pas à définir la science contre des savoirs tels 
que la psychanalyse en neurologie, ou le marxisme dominant dans les sciences sociales et politiques (sous la forme de l'« austro-marxisme" notamment chez Otto Bauer). Paradoxalement, la capacité de ces théories à tout expliquer, tout justifier et à jamais se trouver apparemment prises en défaut, manifeste que, non testables, elles se révèlent invérifiables, puisqu'il est toujours possible de leur trouver quelque légitimation. Popper démarque ces discours de ce qui est proprement l'expression des connaissances scientifiques. Or sa position, que marquait Logik der Forschung en 1929, est l'aboutissement de l'évolution d'un demi-siècle de réflexion de fond qui a mené les sciences naturelles et les sciences sociales à rivaliser de scientificité et à exiger l'apparition, comme juge de paix, pour ainsi dire, d'une nouvelle théorie de la connaissance.

C'est donc sur le fond d'un contexte éminemment propre à développer les antagonismes entre les tendances historicistes (et souvent traditionalistes) et innovantes (et souvent avides d'une simplification purificatrice), que s'inscrit la tentative formulée dans les sciences sociales et en économie politique en particulier, par la perspective qu'ouvre Menger. Celui-ci renouvelle la théorie de la valeur en définissant une alternative, dite marginaliste, à la fois au libre-échangisme britannique et au socialisme académique (Kathedersozialismus) allemand, les deux positions alternatives alors dominantes. Menger n'inaugure pas seulement une ère nouvelle en économie politique avec ses Principes d'économie politique (Grundsätze der Volkswirtschaftslehre, 1871), mais aussi un nouveau chapitre dans la recherche sur la méthode dans ces domaines avec ses Recherches sur la méthode (Untersuchungen über die Methode der Socialwissenschaften [sic] und der Politischen Ökonomie insbesondere, 1883).

Dans le sillage des nouvelles découvertes des sciences naturelles, qui ont influencé de manière décisive la philosophie traditionnelle, toutes les disciplines à prétention et/ou à vocation scientifique - les deux se distinguant au terme d'un demi-siècle de débats dans la stratégie de démarcation qu'allait proposer Popper - se sont donc trouvées obligées d'élaborer des conceptions aussi neuves dans les sciences que leurs analogues dans les arts. Ces vues nouvelles allaient d'une critique de la connaissance à une critique du langage et de la société et elles aboutissaient à réclamer une philosophie anti-métaphysique, empirique et rationaliste. Ernst Mach, Ludwig Boltzmann, Otto Neurath et Ludwig Wittgenstein devaient influencer l'avenir des sciences et le «Cercle de Vienne» (Wiener Kreis) venir à son tour, dans les années 1930, soutenir l'avancée d'une Erkenntnislehre renouvelée.

Le positivisme logique et l'analyse du langage se sont enfin étendus, par la suite, de Vienne vers les universités de langue anglaise, là où les travaux novateurs de Popper retrouvaient, comme Misère de l'historicisme l'illustre éminemment, l'inspiration et les thèmes d'origine des œuvres de Menger. Représentant d'un certain libéralisme, Menger a apporté sa contribution à l'essor d'une culture rationaliste, et à ce titre laïque, et foncièrement innovante. Comme dans les expositions artistiques et comme au théâtre, l'université devait devenir accessible à tous et le savoir qui y était représenté devait pouvoir devenir la propriété commune de tous les citoyens éclairés. C'étaient bien les "Lumières ", d'abord connues sous Joseph II en Autriche, qui semblaient trouver, au moins dans les sciences de la «Vienne 1900 », un nouvel éclat. 


\section{La Vienne de la misère, également source de réflexions économiques}

Pour Menger, l'économie politique est la science des notions économiques "pures ». À ce titre, elle devient un instrument de recherche efficace promis à sortir de l'impasse où l'opposition entre libre-échangisme britannique et socialisme étatiste allemand l'enferme. L'école autrichienne que fonde Menger tranche avec le savoir " historiciste » de l'école allemande dirigée depuis Berlin par Gustav von Schmoller, chef de file de l'université prussienne et grand adversaire de Menger. Leur dispute, le fameux Methodenstreit de la décennie 1880, laisse des traces profondes dans la méthodologie scientifique. Il ne faudrait cependant pas tirer pour autant de cet affrontement long et pénible l'idée que Menger ne se serait pas soucié des faits économiques. Au contraire, il cite la formule de Jean-Baptiste Say selon laquelle « les faits sont nos maîtres à tous ${ }^{4}$ ».

Menger réprouve cependant la méthode inductive qui entend dériver des lois depuis les faits, alors que les lois sont le fait de la théorie pure, et que les faits sont construits par l'esprit sur la base des concepts fondamentaux permettant la production des théories. Précisément, c'est pourquoi Menger manifeste un intérêt constant pour la réalité qu'il a pu observer comme journaliste, puis comme conseiller impérial, qu'il a constaté dans la réalité comme dans les ouvrages de l'énorme bibliothèque (de 20000 volumes) qu'il s'est constitué, et qui contient récits de voyage, rapports administratifs et autres comptes rendus des réalités effectives de Vienne et d'ailleurs.

Alors que les artistes et les écrivains originaires de l'aristocratie et de la grande bourgeoisie mentionnés plus haut comme appartenant à la Jung Wien créaient ce qu'on appela la "littérature fin de siècle", en Autriche il appartenait aux scientifiques de montrer le réel sans ignorer ce qui ne faisait pas partie de leur vie quotidienne. Alors qu'en France, Zola et d'autres auteurs « naturalistes » traitaient de la misère, à Vienne, dans la "littérature fin de siècle" il manquait un regard sur cette autre Vienne qui existait pourtant, à la périphérie, et dont la réalité n'avait point la même brillance, loin s'en fallait. Les différences entre classes sociales étaient considérables, la plus extrême pauvreté voisinait avec une prodigieuse richesse accumulée dans la capitale de l'Empire. Or les disparités n'étaient pas réduites par un essor économique réel, mais partiel et dont le coût était supporté par la couche de la population que le marxisme désignait techniquement, mais en conformité avec la réalité, comme un «esclavage salarié ». La coupure entre ces mondes était telle que l'un paraissait étrange et incompréhensible à l'autre.

Alors les uns souffraient de neurasthénie, les autres mouraient de faim et de froid. Les premiers connaissaient la "maladie fin de siècle » souvent diagnostiquée par Freud ainsi le jeune dandy Anatol, que met en scène Schnitzler, entend se distinguer par sa maladie et il vante sa souffrance qui le distingue de la masse: «Es gibt so viele Krankheiten und nur eine Gesundheit. Man muss immer genauso gesund sein wie jeder andere man kann aber ganz anders krank sein wie jeder andere ${ }^{5}$.» Pendant ce temps, les ouvriers de l'industrie ou du petit artisanat restaient totalement dépendants de leur seule résistance physique pour survivre. Pour construire les monuments somptueux de la Ringstrasse, il avait fallu des masses de travailleurs qui vivaient dans une profonde misère : ceux de l'usine de briques Am Wienerberg (une colline de la banlieue sud de Vienne) peuvent servir d'exemple pour illustrer la condition dans laquelle vivait une partie de la population viennoise. Wolfgang Maderthaner et Lutz Musner écrivent : «In 
dieser Gegend scheint nie die himmlische Sonne. Wiener Vorstädte ${ }^{6}$.» Arrachés par la misère à leur terre d'origine (Bohême et Moravie, dans ce cas), ces travailleurs devaient travailler et vivre dans les pires conditions, hommes, femmes et enfants dormant dans des baraquements jusqu'à soixante-dix par chambrée, car l'entreprise ne fournissait pas de meilleurs logements. Le travail durait quinze heures par jour, sept jours par semaine, leur minime salaire était encore amputé par la pratique des tickets d'approvisionnement qui permettait encore de profiter d'eux (payés en petites plaques de métal, ils ne pouvaient échanger ce salaire que contre les aliments coûteux des cantines de l'usine).

L'expérience de la misère n'épargnait pas les ouvriers qui travaillaient chez de petits artisans. Livrés à l'arbitraire de ces petits patrons, eux-mêmes menacés par la grande industrie, ils trimaient pour des salaires misérables, sans compter que les mauvais traitements n'étaient pas rares. La maladie et la mort étaient omniprésentes dans les quartiers ouvriers de la périphérie viennoise, Simmering, Ottakring et Hernals. Aucune protection sociale ne garantissait les ouvriers contre la maladie ou les hivers rigoureux, aucune allocation en cas de crise économique ne venait soulager la misère de nombreux chômeurs littéralement condamnés à mort. Bien que les licenciements pour activité politique fussent à craindre, les ouvriers, conscients de l'immense injustice sociale qui leur était faite, montraient leur volonté d'agir. Aussi les élections amenaient-elles au pouvoir des partis se disant révolutionnaires, mais aussi des partis apposant le terme "social » à d'autres idéaux culturellement ancrés dans la population, comme le Parti chrétien-social. Ces derniers avaient leur idole dans le leader qu'était Karl Lueger ; à sa mort, ils perdirent nombre d'adhérents et le Parti social-démocrate progressa considérablement. Leur chef, le cofondateur de ce parti en 1888, Victor Adler (né en 1852 à Prague et mort en 1918 à Vienne), avait la confiance et l'adhésion d'une masse de travailleurs salariés. La révolution semblait approcher, au moins dans les discours? ${ }^{7}$.

Divers mouvements socialistes avaient commencé de se manifester dès la révolution de 1848. Le développement de l'industrie, particulièrement en Bohême, et l'augmentation de la population ouvrière vivant dans la misère en conséquence conduisirent à voir naître les premières associations ouvrières, d'ailleurs davantage influencées par le socialisme allemand et les idées de Ferdinand Lassalle que par celles de Karl Marx. Avec la scission entre la tendance libérale (die Gemäßigten) et la tendance marxiste (die Radikalen), le rejet du parlementarisme émergea. Victor Adler réussit à concilier ces deux tendances au Congrès fondateur de Hainfeld (1888). Leader incontesté du Parti social-démocrate d'Autriche (SPÖ) jusqu'à sa mort en 1918, Adler chercha des solutions politiques qui respectaient les exigences sociales au regard de la culture de chaque nationalité de l'État impérial multinational. Sous sa direction, au congrès socialdémocrate tenu en 1889 à Brno, un programme fut élaboré qui donnait aux nationalités la chance d'un dialogue et d'une entente. Devenu en 1905 député du parlement à Vienne, Adler fut un des principaux promoteurs du suffrage universel, qui fut acquis en 1907.

29 C'est dans le cadre de ces décennies de conquêtes sur fond de misère sociale que la fratrie Menger devait illustrer les enjeux de la vie économique autrichienne. Outre Carl, Max, l'aîné, fut un entrepreneur et un député du Parti libéral-national. Il représentait la classe montante innovatrice, tandis qu'Anton, le cadet des trois frères, juriste de formation et de penchant socialiste, devint connu comme l'auteur du premier droit de la consommation impérial et d'un ouvrage bientôt traduit à l'étranger sur le droit des 
travailleurs à recevoir le produit intégral de leur travail. Les thèses du socialisme d'Anton Menger l'éloignaient toutefois de Marx (comme de Rodbertus, sans doute moins de Lassalle) ${ }^{8}$.

L'écho des thèses universitaires ne parvenait certes que très assourdi dans les congrès ouvriers, si grands que fussent les efforts de l'appareil du parti organisé par Adler pour assister les masses dans les luttes pour obtenir des droits. En même temps, les thèses socialistes réformistes comme celles d'Anton Menger contribuaient à prévenir le déclenchement d'une révolution. Les radicaux, proches des sociaux-démocrates russes, ne pardonnaient à Adler sa politique attentiste (Politik des Abwartens) qu'en raison de ses effectifs nombreux succès en pratique.

31 C'est à cette époque que la Seconde Internationale (socialiste) fut fondée à Paris en 1889, à l'initiative de Friedrich Engels. Elle trouva en Adler l'un de ses membres les plus influents. Le Parti social-démocrate touchait alors toute la classe ouvrière de l'empire habsbourgeois à travers ses organisations : fondée en 1894, «l'Association des jeunes travailleurs" (Jugendliche Arbeiter) motivait la conscience de classe des plus jeunes; le mouvement pour une "École populaire " (Volksbildungsverein Ottakring) allait à terme nourrir des projets de réforme pédagogique majeure (Karl Bühler, Otto Glöckel) ${ }^{9}$; les bibliothèques populaires, les loisirs peu coûteux au sein de la nature (le club Naturfreunde), les activités sportives, comme le cyclisme et les randonnées, de nombreux clubs de gymnastique, notamment, devaient renforcer l'esprit et le corps des travailleurs. Ce travail éducatif et culturel fut réalisé par "das rote Wien », la capitale d'un Empire qui allait tomber, mais dont le modèle social devait perdurer aux yeux des partisans modérés d'une transformation sociale graduelle au sein de la Seconde Internationale.

Carl Menger publia plusieurs articles au sujet des réformes sociales de son temps dans le journal Neue Freie Presse, notamment dans l'édition des 6 et 8 janvier 1891, intitulé "Die Social-Theorien der classischen National-Ökonomie und die moderne Wirtschaftspolitik ${ }^{10} »$. Menger y écrit en économiste, à la différence de son frère cadet qui raisonne toujours en juriste historiciste, avec quelques conséquences dans le manque de compréhension dans leurs relations. Menger souligne la position critique des pères de l'économie politique contre les dépenses excessives de l'État, et en cela il ne partage pas les convictions de son cadet. C'est toutefois autant par prudence que parce que le rôle du savant n'est jamais selon Carl celui d'un avocat, sa cause fut-elle la meilleure, que Menger souligne que la neutralité doit rester l'apanage de la science. Il comprend avant tout le devoir du scientifique comme celui d'éclaircir les débats de son temps sans jamais subordonner la science ni la mettre au service d'une opinion ou d'un mouvement politique, quel qu'il soit. L'intégrité intellectuelle doit conduire à repousser les bavardages des "avocats " (Advokaten) des causes politiques ${ }^{11}$. C'est aussi pourquoi Menger évite toute prise de position publique en faveur d'un parti. En cela, il se différencie de son frère aîné Max, qui s'est fait élire député. Pour Carl, il en va de la théorie même et, si pratique que puisse être le but ultime de la science, en particulier dans les sciences sociales et en économie, celle-ci doit rester neutre axiologiquement (wertfrei).

En conséquence, selon Menger, ni communisme, ni socialisme, ni libre-échangisme ne sont des positions à partir desquelles élaborer une théorie adéquate. L'exigence d'une science indépendante est de ne pas partir de telles positions, mais d'élaborer un savoir qui, possiblement, déduis de ses connaissances avérées des cas où telle ou telle 
politique peut s'avérer la meilleure en pratique selon les conditions du moment. Toute autre attitude consiste à servir des causes et en outre les deux premiers courants cités plus haut utilisent par construction des concepts d'entités collectives (Kollektivbegriffe) dont l'existence est postulée ontologiquement sans moyen de prouver sa pertinence méthodologique. La réalité des actions individuelles économiques (Privatwirtschaften) doit conduire à la prudence quant à l'ontologie et à l'individualisme méthodologique. À l'idée d'une économie collective à la base de leur théorie, Menger oppose donc l'analyse des économies "privées", puisque c'est toujours en observant du point de vue de la méthode l'individu comme tel que l'on peut analyser ses décisions. Cet individualisme méthodologique, qui devait devenir la marque de l'école "autrichienne", est le pendant d'une reconnaissance scientifique de la subjectivité de l'individualité dans les sciences sociales et en économie politique en particulier.

\section{L'obstacle majeur : le mythe habsbourgeois de la coexistence harmonieuse des nationalités}

\section{Luttes d'influence à travers les langues nationales}

Il convient de saisir, pour finir, quant au contexte de l'enseignement des sciences économiques dans l'empire austro-hongrois, les enjeux des disputes concernant les nationalités et les langues d'enseignement. Menger écrit évidemment en allemand. Mais la situation, dans les universités mêmes où il a étudié, n'est pas toute simple. Il convient de l'éclaircir pour le lecteur.

La réforme de Léo Thun-Hohenstein (qui était ministre de l'Éducation dans les années 1850) permit d'en finir avec un immobilisme séculaire à l'université de Vienne quant au recrutement des professeurs en accordant une autonomie toute nouvelle à l'université dans les domaines de la recherche et de l'enseignement. Menger en profita lui-même et l'«École autrichienne d'économie » prit corps dans cette perspective.

L'autonomie venait cependant après des conflits nombreux. Surtout, cette école héritait de ces derniers. On en évoquera un ici que Menger a connu, car il a fréquenté des cours allemands et des cours tchèques à l'université de Prague. Il a su nouer des contacts avec des collègues tchèques, et comprendre leurs revendications nationales. Plus tard, il devait intervenir pour débloquer des ressources financières pour l'enseignement supérieur à l'université de Prague. Si l'on veut comprendre les enjeux de ces questions, il convient de replacer, même brièvement, cette lutte dans son contexte.

Joseph II (empereur de 1780 à 1790), nous le rappelions, a présidé aux premières «Lumières » en Autriche, à la fin de l'époque correspondante en France, qui s'achevait certes dans les troubles révolutionnaires. Joseph II a instauré une langue unique, l'allemand, comme langue de l'administration et de l'enseignement. La mesure, qui se voulait modernisatrice, frustra les esprits nationalistes et elle éveilla leur résistance. Dans l'espace de l'Empire cohabitaient en effet assez de nations différentes pour parler neuf langues vernaculaires (au moins): l'allemand, le tchécoslovaque (si l'on ne distingue pas le tchèque et le slovaque, ce qui est encore une autre question), le polonais, le ruthène, le slovène (la langue de la province alors appelée Carniole), le serbo-croate (là encore, si l'on ne distingue pas), l'italien-ladin (la même remarque s'imposant à une époque où l'italien est concurrencé partout par les patois, au moins 
dans la langue courante), le roumain et le hongrois. Ainsi, au moins neuf langues (si l'on ne les subdivise pas elles-mêmes comme le tchèque et le slovaque, le serbe et le croate, etc.) se partageaient un territoire où les zones de contact entre cultures et traditions distinctes devenaient autant d'espaces potentiels de confrontations et de conflits.

Le compromis austro-hongrois conclu en 1867 avait divisé l'Empire en deux parties : la Transleithanie (royaume de Hongrie) et la Cisleithanie (toutes les autres régions, selon l'expression alle anderen im Reichsrat vertretenen Königreiche und Länder) également englobées dans ce qui était alors appelé «Autriche». Cis- et Transleithanie étaient unies par l'union personnelle avec François-Joseph I, empereur d'Autriche et roi de Hongrie (d'où le fameux acronyme k.u.k. pour kaiserlich und königlich). Chaque partie avait sa propre constitution, son Parlement et ses Premiers ministres. La politique étrangère, les finances et l'armée restaient communes. Cette décision qui mettait en place un État bicéphale, avec Vienne et Budapest comme capitales, confirmait, dans l'opinion des autres nationalités, la domination des Allemands et des Magyars.

39 La situation s'aggravait, ici ou là d'un ressentiment local, par exemple en Bohême et en Moravie $^{12}$, où les Tchèques se sentaient discriminés, car ils n'avaient pas eu de concessions dans le compromis de 1867. Ils étaient dans l'impossibilité d'imposer la reconnaissance de leurs droits dans un État «à trois », et ce malgré le loyalisme certain qu'ils continuaient de témoigner envers la dynastie des Habsbourg. Or, après 1867, les germanophones représentaient $37 \%$ de la population de Cisleithanie, une situation démographique qui faisait craindre aux Allemands de perdre leur influence politique. Pour prévenir tout risque de dissolution, une politique volontariste vint régler l'enseignement et l'apprentissage des principales langues dans l'Empire.

Dans la Constitution de décembre 1867, l'article 19 garantit le principe d'égalité de droits aux langues de Cisleithanie : chacun doit être instruit, formé et administré dans sa langue maternelle; le $\$ 3$ interdit de rendre obligatoire pour quiconque d'apprendre une deuxième langue. Là où vivaient plusieurs nationalités, les institutions scolaires publiques auraient donc dû être organisées de façon à ce que personne n'ait à apprendre de deuxième langue, chacune des nationalités recevant dans sa langue les moyens nécessaires à sa formation.

41 Cet article eut des conséquences lourdes : les Allemands se montraient réticents à l'idée que le tchèque, qu'ils maîtrisaient rarement, pût devenir langue administrative en Bohême, et les Tchèques en retour s'appuyaient sur l'article 19 pour obtenir des ordonnances allant dans ce sens. Lorsqu'il fut ministre de l'Enseignement, Karl von Stremayr fit en conséquence imposer le bilinguisme aux fonctionnaires en contact avec les administrés. Les germanophones se refusaient toutefois à abandonner leurs bastions dans l'administration, les Tchèques insistèrent et les décrets de Casimir Badeni (ministre-président en 1897) rendirent obligatoire la maîtrise du tchèque et de l'allemand à tous les fonctionnaires de Bohême dans la correspondance administrative interne comme externe, ce qui déclencha une violente crise monarchique - il va de soi que les germanophones connaissaient moins souvent le tchèque que leurs collègues de langue tchèque l'allemand, et les positions dans l'administration étaient recherchées, parfois comme des sinécures où la fameuse Schlamperei pouvait se donner libre cours ${ }^{13}$. Sous la pression, les gouvernements suivants allaient devoir retirer progressivement ces ordonnances. 


\section{Le déséquilibre économique entre les régions centrales et périphériques de l'Empire}

Les luttes d'influence à travers la langue recoupent évidemment un autre décalage majeur, d'ordre socio-économique. Le compromis passé en 1867 impacte fortement l'économie comme la politique de la Cis- et de la Transleithanie, bref de l'Empire ${ }^{14}$. Sans pour autant être administrés en commun, certains domaines-clés (la dette de l'État, le commerce, la fiscalité et l'administration des douanes et des transports) concernent ses deux parties. La question centrale est posée par la "Quote ", la charge financière que chaque royaume devait accepter de prendre en charge dans le budget commun. L'accord se fit sur un rapport de $70 \%$ pour l'Autriche et $30 \%$ pour la Hongrie, mais la question demeura un point de friction permanent entre Vienne et Budapest jusqu'à la fin de la monarchie.

Malgré les difficultés économiques héritées du passé et les contraintes nouvelles imposées aux deux entités, les premières années après le compromis sont marquées par une croissance économique remarquable, avec une période de forte hausse (1867-1873) interrompue par la Grande Dépression (1873-1890) et un redressement économique effectif (1890-1914). Durant les sept années qui précèdent le krach boursier (Gründerkrach) de 1873, l'Autriche-Hongrie expérimente une politique de redressement et de rationalisation économiques facilitée par la politique déflationniste mise en œuvre à partir de 1860 et par la forte circulation monétaire qui suivit l'abolition des restrictions au libre-échange entre les provinces dès 1865.

Jusque-là, pour pallier l'inconvénient d'avoir encore des provinces assez cloisonnées, l'État aurait dû faire de grands investissements, mais les moyens financiers lui ont manqué en raison, d'une part, des dépenses militaires et, d'autre part, d'un système politique qui privilégiait les intérêts de l'aristocratie foncière (surtout), des banquiers, des grands négociants (dans les grandes villes) et des industriels déjà établis (dans des centres encore trop rares et très concentrés). En outre, un statut et un règlement propres à chaque province, outre une politique d'État trop inconstante, ont pénalisé les régions éloignées du centre, qui restaient exclues de la possibilité de vendre leurs produits à bon prix. À la suite du compromis de 1867, l'État réduit ses dépenses militaires et encourage le redressement économique : on assiste à un doublement du réseau ferroviaire entre 1865 et 1870 en Autriche et en Hongrie, ce chiffre à lui seul est significatif du mouvement qui avait été enclenché.

Or, les frères Menger, nés en Galicie, ont pu dans leur enfance observer de près la souffrance et la vie misérable des paysans dépendants des grands propriétaires. Devenu précepteur du jeune prince héritier, Menger voulut contribuer à améliorer cette situation déplorable. Pour montrer au Kronprinz les effets de la modernisation industrielle et économique, Menger accompagne Rudolf lors de son «Grand tour » de l'Europe de l'Ouest : ensemble ils visitent l'Allemagne, la Suisse, la France et la GrandeBretagne. Impressionné par ce qu'il y a vu, le prince peut comparer la situation économique des pays avancés et celle des zones rurales arriérées dans sa patrie. Une cause était, selon lui (et sous l'éclairage et le pilotage de son mentor), la résistance d'une noblesse rurale ennemie de toute modernisation qui lui ôterait des privilèges ancestraux. À son retour, le jeune prince rédige un article, qu'il fait publier avec l'aide de Menger, de manière anonyme, chez un éditeur allemand à Munich. Dans cet article intitulé «La noblesse autrichienne et sa vocation constitutionnelle ${ }^{15}$ », le prince 
héritier dirige ses attaques contre l'incurie et l'obscurantisme des jeunes aristocrates uniquement préoccupés de chasse et de parades militaires. Leur manque de participation à la vie parlementaire, leur désintérêt pour les résultats des études scientifiques, économiques et politiques freinent tout développement dans la valorisation de leurs propriétés et l'amélioration des conditions de vie de la population rurale. Il le leur reproche et il prône une ouverture, notamment en direction des classes entreprenantes de la bourgeoisie.

La justesse de ces observations se vérifia de la période d'expansion économique à celle de la crise économique qui devait durer de 1873 à 1890, avec un impact profond sur la mentalité des populations de l'Empire, car elle détruisit la confiance en une prospérité durable et prépara la place à des sentiments haineux de rejet et d'exclusion, d'une part des classes défavorisées vis-à-vis des classes possédantes, d'autre part, des populations nationales entre elles; en particulier, la haine des Juifs (accusés d'être responsables de la crise et de faillites) s'accrut énormément avec la chute mémorable d'une banque non-juive comme la Credit-Anstalt.

Malgré la "Grande récession », la double monarchie continue de connaître de manière générale une expansion presque continue, tout spécialement dans les secteurs de l'industrie (les machines-outils, par exemple, avec des taux de croissance annuels de $2 \%$ en Autriche et de 3,5\% en Hongrie). Par un effet de contrecoup, le monde agricole subit les répercussions négatives de la crise et il doit se contenter d'une position retardataire, notamment par rapport aux progrès obtenus par certains ouvriers et employés dans les villes. Une grande partie de la population rurale reste sans emploi ni qualification, ce qui cause un fort exode rural (Landflucht) vers ces villes, amplifiant le processus d'urbanisation jusqu'en 1914 : à Vienne, la population quadruple entre 1850 et 1914, pour atteindre alors plus de 2 millions d'habitants.

Au bout du compte, l'économie de l'Empire connaît entre 1870 à 1910, à travers l'expansion, la crise et le redressement, une phase de mutation industrielle assez rapide. La modernisation de l'agriculture reste très variable selon les régions, et la disposition des propriétaires locaux à introduire ou pas, des innovations techniques et des progrès dans l'éducation rurale. L'achèvement du réseau ferroviaire jusqu'aux frontières orientales de la Hongrie joue un grand rôle. Au tournant du $\mathrm{xx}^{\mathrm{e}}$ siècle, Vienne, assurément grande métropole européenne, trouve toutefois un pendant dans l'autre capitale de la Double Monarchie, Budapest, elle aussi devenue une grande ville de la culture et de la politique.

Néanmoins, même ces capitales ne sont souvent qu'une étape dans un phénomène de migration volontaire plus large qui entraîne, vers les États-Unis et le Canada, des centaines de milliers de Slaves, de Hongrois ainsi qu'une forte minorité juive (également chassée des territoires de l'Est par la vive haine antisémite qui y règne). Malgré les efforts réalisés pour unifier les territoires de l'Empire par le libre-échange et le développement des infrastructures de transport, les profondes inégalités entre provinces ne devaient pas être corrigées avant la Grande Guerre ${ }^{16}$. Ensuite, l'empire n'existait plus et les destins de ces nations nouvelles allaient varier. On peut dire qu'aucun changement profond n'a vraiment eu lieu, en particulier dans les structures de la propriété foncière en Hongrie et en Bohême au cours du demi-siècle suivant le compromis de 1867.

Les classes possédantes attachées à leurs privilèges s'opposent victorieusement au progrès économique et technique; les grands propriétaires continuent d'exercer une 
domination souvent crasse. En outre, sous la Monarchie, les postes de la haute-fonction politique restent en général réservés aux aristocrates : après leur formation, ceux-ci assument des postes de responsabilité et ils refusent les idées libérales et nationales qu'ils tiennent pour dangereuses (non seulement pour leur propre position, mais aussi pour l'unité de la Monarchie, ce qui n'était pas erroné). La politique menée pendant cette période peut en conséquence être qualifiée à juste titre par un constat: flottements, hésitations et propension à prendre les décisions en faveur des régions plus avancées.

51 Le prince Rudolf lui-même, malgré l'influence positive de son mentor Menger, fut découragé et déprimé par l'attitude de son père, l'empereur François-Joseph, et surtout par celle de son entourage à la Cour. En se suicidant en 1889, il mit fin à l'espoir d'un avenir plus éclairé. Des réformes réelles, comme le changement monétaire de 1892 (Valutareform) auquel Menger participa activement, eurent des effets positifs (ainsi sur le redressement économique postérieur à la crise). Elles ne changèrent pas pour autant la tendance profonde et l'intervention de l'État, même lorsqu'elle était positive (ce qu'un disciple libéral de Menger, Eugen von Böhm-Bawerk, devenu ministre, était prêt à reconnaître), ne pouvait compenser ni les faiblesses inhérentes au bas niveau de développement dans les provinces, quelque important que fût l'élan donné à leur modernisation, ni l'accumulation insuffisante de capital productif dans les centres impériaux, aussi massif que pût paraître l'effort de capitalisation bancaire à différents moments (ce qui suggéra à Karl Hilferding, austro-marxiste quant à lui, l'idée du « capital financier » comme stade de l'industrialisation).

\section{Conclusion}

Presque tous les ouvrages sur la «Vienne fin de siècle » étudient et illustrent l'essor intellectuel, économique et artistique de la capitale autrichienne. Nous avons voulu montrer en quoi cette toile de fond doit être couplée avec celle de la situation socioéconomique et comment, de la sorte, essor intellectuel et conscience du réel se conjuguent pour donner une science économique renouvelée. Dans Le monde d'hier. Souvenirs d'un Européen, Stefan Zweig s'est fait le chroniqueur de l'âge d'or vécu par l'Autriche habsbourgeoise : «Arm und reich, Tschechen und Deutsche, Juden und Christen lebten trotz gelegentlicher Hänseleien friedlich beisammen ${ }^{17}$." Ces souvenirs personnels datent d'une époque où la monarchie des Habsbourg n'existait cependant déjà plus : Zweig vivait alors en exil loin de sa patrie. Une raison est que la réalité avait été en partie différente de l'âge d'or rêvé rétrospectivement.

À la veille de la Grande Guerre en 1914, l'empereur François- Joseph, alors âgé de 84 ans, s'adressa à ses sujets dans une lettre ouverte «à ses peuples » (An meine Völker). La formulation montre clairement, par son déni de la réalité, que le rêve de l'union des peuples était en réalité mort depuis longtemps. Malgré les luttes d'influence (et les violences) entre nationalités, l'allemand est resté la langue officielle de l'Empire et les institutions chargées de former les hauts fonctionnaires, les diplomates et les officiers (avant tout la Militärakademie de Wiener Neustadt, le Theresianum et la Diplomatische Akademie à Vienne) enseignaient en allemand, et cela allait de soi. La suspension de la Diète de Prague (la Cour provinciale suprême de la Bohême) en juillet 1913 devait mettre un point final à la période d'agitations et de désordre qui avait caractérisé la vie 
parlementaire - mais aussi aux tentatives de conciliation entre Allemands et Tchèques...

Ni les solutions politiques, qui n'en étaient pas vraiment au final, ni les politiques économiques qui, menées de Vienne et de Budapest, n'atteignaient pas les provinces ne pouvaient aplanir les disparités de développement entre les régions de l'Empire. Concernant les réformes, parfois tentées et si peu souvent abouties, si elles parvenaient, dans de rares cas, à moderniser des pans de l'économie (comme le fit la Valutareform de 1892 pour la monnaie), le succès dépendait directement de la bonne volonté et des choix économiques du gouvernement à Vienne. Quels que fussent les efforts de la part des provinces, la résolution à traiter équitablement (ou non) les diverses parties de l'Empire se prenait (ou pas, le plus souvent) à Vienne.

Jusqu'en 1914, la relative inégalité qui existait entre les provinces ne fut pas modifiée, non seulement parce que le dualisme du compromis austro-hongrois de 1867 avait favorisé les provinces autrichiennes au détriment du reste de l'Empire sur les plans commerciaux, fiscaux et budgétaires, mais encore parce que le manque de ressources affectait les provinces périphériques qui étaient déjà les moins capables d'autosuffisance. Les écarts moyens de richesse allaient encore à bien plus de trois contre un entre la province la plus riche de Basse-Autriche (celle qui comprend Vienne) et la plus pauvre (la Bucovine, tout à l'Est). Malgré un rattrapage parfois considérable dans certains secteurs pour les régions les plus arriérées, le décollage économique affectait seulement certaines zones ou certains secteurs. On peut mesurer au pourcentage de population agricole l'état de transformation et de développement socio-économique des régions : en 1910 à Vienne et en Basse-Autriche il était de $20 \%$, en Bohême et en Moravie de $40 \%$, en Hongrie de $70 \%$, et en Galicie, d'où venait Menger, ou en Bucovine de presque $80 \%^{18}$. Il faut garder ce facteur territorial en tête lorsqu'on évalue l'influence de l'école autrichienne fondée par Menger: elle appartenait déjà au $\mathrm{xx}^{\mathrm{e}}$ siècle par ses conceptions alors que l'Empire vivait encore au $\mathrm{XVIII}^{\mathrm{e}}$ siècle dans de nombreuses régions. Tant d'autres causes jouèrent évidemment, mais un empire tel que l'Empire habsbourgeois devait périr. L'espoir que recélaient les théories de l'école autrichienne d'économie se trouvait fondé sur la pensée innovante de Menger : sinon l'empire, du moins les idées pouvaient survivre.

\section{NOTES}

1. Carl Menger, Gesammelte Werke, Friedrich A. Hayek (éd.), Tübingen, J. C. B. Mohr, 1968-1970, p. XII.

2. Parmi les nombreuses publications qui existent sur ce thème, nous voulons mentionner Carl $\mathrm{E}$. Schorske, Vienne fin de siècle. Politique et culture, Paris, Seuil, 1983.

3. Il devint le créateur de la maison de Michaelerplatz (connue sous le nom de «maison Loos »: Looshaus) qui, avec ses fenêtres sans décor (qui valurent à l'édifice le surnom de la « maison sans cils ») et ses murs nus, contraste violemment avec l'imposante résidence impériale de la Hofburg. Cette maison (initialement la maison de couture Goldman \& Salatsch) devint un véritable objet de 
scandale pour la bourgeoisie traditionaliste qui était au contraire enthousiasmée par l'éclectisme décoratif du Ring.

4. Concernant la relation de Menger à Say, voir notamment Gilles Campagnolo, Critique de l'économie politique classique. Marx, Menger et l'École historique, Bernard Bourgeois (préf.), Paris, PUF, 2004, chap. 8.

5. Arthur Schnitzler, Die dramatischen Werke. Bd. I., Frankfurt/M., S. Fischer, 1962, p. 84.

6. Michael Schwarz (éd.), Ganz unten. Die Entdeckung des Elends, Wien, Brandstätter, 2007.

7. Helene Maimann, Die ersten hundert Jahre. Österreichische Sozialdemokratie 1888-1998, Wien, Brandstätter, 1988.

8. Sur la fratrie Menger, et les éléments biographiques évoqués ici, voir le seul ouvrage disponible en français: Gilles Campagnolo, Carl Menger, entre Aristote et Hayek. Aux sources de l'économie moderne, Paris, CNRS, 2008.

9. Sur la réforme de l'école par le travail (Arbeitsschule) et le mouvement pédagogique de cette époque, à travers les écrits d'un jeune philosophe réformiste, Popper, voir Karl Raimund Popper, Apprentissage et découverte. Écrits de jeunesse, Vienne 1925-1935, Gilles Campagnolo (trad. et éd.), Paris, Rue d'Ulm, «Versions françaises », 2019.

10. Erich Streissler, «Carl Mengers Vorlesungen aus politischer Ökonomie für Kronprinz Rudolf », dans Michael Benedikt (dir.), Über Gesellschaft hinaus, Wien, Leben-Kunst-Wissenschaft, 2000 , p. 55.

11. Menger porta en note sur la page intercalaire de son volume personnel de ses Grundsätze de 1871: «Dass pol[itische] Econ. eine ganz neutrale wissenschaft ist, weder Kathedersoci[alistisch] noch Freihändler, noch communistisch.» Une autre citation porte: «Communists, Socialists of the Chair, partisans of Free-trade, all advocates! (Kommunisten, Kathedersozialisten, Freihändler, alle Advokaten!) Voir Gilles Campagnolo, Critiques de l'économie politique classique, op. cit.)

12. Gerhard Stourzh, «Die Völker des Reiches» dans Adam Wandruszka et Peter Urbanitsch (dir.), Die Habsburger Monarchie, 1848-1918, Wien, Verlag der österreichischen Akademie der Wissenschaften, 1980, t. III.

13. Hannelore Burger, Sprachenrecht und Sprachengerechtigkeit im österreichischen Unterrichtswesen 1867-1918, Wien, Verlag der österreichischen Akademie der Wissenschaften, 1995, p. 80 et suiv.

14. Roman Sandgruber, Ökonomie und Politik. Österreichische Wirtschaftsgeschichte vom Mittelalter bis zur Gegenwart, Wien, Ueberreuter, 2005, Bd. X.

15. Anonyme reconnu comme étant le Kronprinz Rudolf, "Der österreichische Adel und sein constitutioneller Beruf», dans Brigitte Hamann, Kronprinz Rudolf, Majestät, ich warne Sie, München-Zürich, Pieper, 1987, p. 20-52.

16. Andrea Komlosy, «Regionale Ungleichheiten in der Habsburgermonarchie. Kohäsionskraft oder Explosionsgefahr» dans Hans-Heinrich Nolte (dir.), Innere Peripherie in Ost und West, Stuttgart, Franz Steiner, 2001, p. 97-111.

17. Stefan Zweig, Die Welt von gestern. Erinnerungen eines Europäers, Frankfurt/M., S. Fischer, 1982, p. 40. Zweig dut fuir en 1934 en raison du nazisme et, peu avant de se suicider (au Brésil, en 1941), il écrivit ce livre-testament.

18. Helmut Rumpler, Eine Chance für Mitteleuropa. Bürgerliche Emanzipation und Staatsverfall in der Habsburger-monarchie, Wien, Ueberreuter, 2005, p. 458. 


\section{RÉSUMÉS}

La Vienne de Carl Menger déployait sa splendeur dans tous les domaines des arts et des sciences. Du fait de ses activités à l'université de Vienne, de son travail pour des journaux et de sa collaboration à des cabinets ministériels, Menger put suivre de près les événements de son époque, y participer et exercer son influence sur la modernisation de l'université de Vienne et sur la politique économique (comme le montra la réforme monétaire de 1892). Dans le champ de l'économie politique, Menger renouvela la théorie de la valeur en définissant l'alternative dite "marginaliste» au libre-échangisme britannique et au socialisme académique en vogue en Allemagne. En raison de ses politiques hésitantes sur la voie de la modernisation menée par ses hauts fonctionnaires, l'Empire des Habsbourg devait disparaître, mais l'« école autrichienne d'économie » fondée par Menger allait en revanche se développer et demeurer jusqu'aujourd'hui. Ce texte présente Menger, fondateur de l'« école autrichienne » en son temps et dans sa ville.

Carl Menger lebte in einer sehr bewegten Zeit. Ab der zweiten Hälfte des 19. Jahrhunderts setzten auf allen Gebieten des öffentlichen und privaten Lebens, der Politik, der Wirtschaft, der Wissenschaft, der Baukunst und der bildenden Kunst gewaltige Auseinandersetzungen und Änderungen ein. In Wien, dem Zentrum der Habsburgermonarchie und Sammelbecken aller Bewegungen, konnte Menger dank seiner akademischen Ausbildung und seiner beruflichen Funktionen einen sehr guten Überblick über die Entwicklung auf allen Gebieten erhalten. Durch sein Wirken in politischen Gremien, an der Wiener Universität und als Beiträger einer qualitativ hochstehenden Tageszeitung konnte er Einfluss auf die Entwicklung nehmen. In seinem wissenschaftlichen Werk, das sowohl einen theoretischen als auch einen empirischen Teil enthält, ohne diese beiden Teile zu vermengen, gelang ihm das, was der Bürokratie des Habsburgerreiches nicht gelungen war: trotz der Beibehaltung von Strukturen Transformationen durchzusetzen. Wenngleich die Habsburgermonarchie aufgrund der Unbeweglichkeit, Halbherzigkeit und Nachlässigkeit ihrer Bürokratie bei der Ausführung notwendiger Reformen dem Untergang geweiht war, lebt das Denken Carl Mengers in der von ihm gegründeten „Österreichischen Schule“ der Nationalökonomie bis heute fort.

Carl Menger lived in a very eventful period in Vienna, the center of the Hapsburg monarchy. From the $2^{\text {nd }}$ half of the $19^{\text {th }}$ century onward, debates on art, architecture, politics, economy and sciences permeated all realms of public and private life. From this melting-pot of peoples and innovative currents, owing to his academic activity Menger got an excellent overall picture of such developments. While working in governmental committees and contributing articles to a quality daily newspaper, he gained influence in economic matters and his scientific work contained both a theoretical and an empirical part so that he brought about changes in spite of conservative structures which the Hapsburg bureaucracy maintained through rigidity combined with half-hearted negligence, while failing to realize full-fledged modernization. The Hapsburg monarchy was thus doomed to decline; conversely, Menger's ideas were furthered by the Austrian School of Economics. This paper introduces the founder of the Austrian School in the context of his times in his city.

\section{INDEX}

Schlüsselwörter : wirtschaftslehre, Menger (Carl), österreichischen schule, Wien

Keywords : Vienna, austrian economic ideas, Menger (Carl), economic thought

Mots-clés : doctrine, Menger (Carl), école autrichienne, Vienne 